Arbeitslosenversicherung

\section{Zugang für alle Selbstständigen}

Die Coronakrise führt den Wert sozialer Sicherung dramatisch vor Augen. Während sozialversicherungspflichtig Beschäftigte durch den Anspruch auf Kurzarbeitergeld und Arbeitslosengeld abgesichert sind, ist das bei Selbstständigen in aller Regel nicht der Fall. Daher sind viele Selbstständige in finanzielle Schwierigkeiten geraten. Aus dem Stegreif mussten Unterstützungspakete geschnürt werden, die aber oft als zu eingeschränkt wahrgenommen werden. Gerade Lebenshaltungskosten - also die Unternehmerlöhne - waren nicht abgesichert, sieht man von der Grundsicherung ab, die jedoch trotz der in der Coronakrise vereinfachten Bezugsbedingungen für viele Selbstständige nicht infrage kommt. Notwendig ist also eine planbare Absicherung von Selbstständigen gegen gravierende Einkommensrisiken.

Derzeit können sich Selbstständige in den ersten drei Monaten ihrer selbstständigen Tätigkeit für die freiwillige Arbeitslosenversicherung entscheiden. Diese Möglichkeit wird auch aufgrund der restriktiven Bedingungen allerdings kaum in Anspruch genommen (Jahn und Oberfichtner, 2020). Auf dem Weg zu einer umfassenderen und effektiveren Absicherung gibt es ein großes Potenzial an Reformen (Schoukens und Weber, 2020). Im Koalitionsvertrag der Ampel wird die Absicht erklärt, den Zugang zur freiwilligen Arbeitslosenversicherung zu erleichtern und zu prüfen, „ob und wie ein Zugang ohne Vorversicherungszeit möglich ist". In der Tat: Für den Eintritt in die freiwillige Arbeitslosenversicherung sind Vorversicherungszeiten erforderlich. Faktisch handelt es sich also nicht um eine Arbeitslosenversicherung für Selbstständige, sondern um eine für ehemalige sozialversicherungspflichtig Beschäftigte.

Im Sinne eines besseren Zugangs wäre es wünschenswert, wenn sich einfach alle Selbstständigen zu einem beliebigen Zeitpunkt versichern könnten. Was könnte problematisch sein? Es bestünde wohl die Gefahr, dass diese Möglichkeit dann gerade diejenigen anzieht, für die eine Geschäftsaufgabe schon absehbar ist. Dem könnte man mit einer grundsätzlichen Prüfung beim Eintritt entgegenwirken. Natürlich verfügt der Selbstständige gegenüber der Arbeitslosenversicherung über asymmetrische Information. Aber eine grobe Verschlechterung der Geschäftslage bzw. -aussicht dürfte dennoch oft mit

(C) Der/die Autor:in 2022. Open Access: Dieser Artikel wird unter der Creative Commons Namensnennung 4.0 International Lizenz veröffentlicht (creativecommons.org/licenses/by/4.0/deed.de).

Open Access wird durch die ZBW - Leibniz-Informationszentrum Wirtschaft gefördert. vertretbarem Aufwand erkennbar sein. Auch wäre zu versichern, dass eine solche aktuell nicht vorliegt, sodass eklatanten Fällen auch im Nachhinein bei Leistungsbeantragung noch nachgegangen werden könnte. Weiterhin könnte man bei nachträglichem Eintritt in die Arbeitslosenversicherung eine relativ lange Anwartschaftszeit vorsehen. Diese würde es unwahrscheinlich machen, dass eine Geschäftsverschlechterung schon absehbar ist. Die Beiträge könnten währenddessen entsprechend niedriger liegen. Eine zweite Gefahr bestünde darin, dass die Arbeitslosenversicherung abgeschlossen wird, um einen baldigen Vorruhestand finanziell abzusichern. Dem könnte aber mit Altersgrenzen begegnet werden. Schließlich ist davon auszugehen, dass etwa jenseits der 60 kaum ein Selbstständiger ohnehin noch ernsthaft in die Arbeitslosenversicherung eintreten wollte.

In einer eingeschränkteren Variante wäre es auch denkbar, die Möglichkeit des jederzeitigen Eintritts nur in einer z.B. zweijährigen Anfangsphase nach Gesetzesänderung zu gewähren. Damit hätten alle jetzigen Selbstständigen die Chance, sich zu versichern, und alle zukünftigen Selbstständigen könnten dies innerhalb einer deutlich erweiterten Frist zu Beginn ihrer Selbstständigkeit tun.

Die Leistungshöhe der freiwilligen Arbeitslosenversicherung ist am früheren Gehalt in sozialversicherungspflichtiger Beschäftigung orientiert, bzw. wenn diese schon länger zurückliegt, steigt sie mit der formalen Qualifikation. Letzteres würde auf alle nachträglichen Einsteiger:innen zutreffen. Ein Grund mehr, Leistungen und Beiträge entsprechend dem Äquivalenzprinzip am laufenden Einkommen zu orientieren (Schoukens und Weber, 2020). Denn momentan sind die Beiträge für alle gleich hoch - Solidarität einmal andersherum. Zudem würden aussichtslose Gründungen mit dem Ziel, Leistungen der Arbeitslosenversicherung zu beziehen, so unattraktiv.

Die hier vorgestellten Vorschläge wären geeignet, im Rahmen des Koalitionsvertrags möglichst große Effekte zu erzielen. Für eine wirklich breite Beteiligung dürften darüber hinaus weitere Schritte notwendig sein, wie zahlreiche internationale Beispiele zeigen.

Enzo Weber Institut für Arbeitsmarkt- und Berufsforschung (IAB)

\section{Literatur}

Jahn, E. und M. Oberfichtner (2020), Freiwillige Arbeitslosenversicherung: Nur wenige Selbstständige versichern sich gegen die Folgen von Arbeitslosigkeit, IAB-Kurzbericht, 11.

Schoukens, P. und E. Weber (2020), Unemployment insurance for the self-employed: a way forward post-corona, IAB-Discussion Paper, 32. 